\title{
Comportamiento métrico sobre evaluación de la educación en emprendimiento
}

\author{
Valeria Cantú González* \\ Leonardo David Glasserman Morales* \\ María Soledad Ramírez Montoya*
}

Artículo recibido:

12 de enero de 2018

Artículo aceptado:

9 de enero de 2019

Artículo de investigación

\section{Resumen}

El presente artículo tuvo como objetivo presentar los resultados de un mapeo sistemático acerca de la evaluación de la educación en emprendimiento. El propósito fue identificar las publicaciones más recientes sobre el tema en cuestión. Se realizó un mapeo sistemático siguiendo la metodología propuesta por Petersen et al. (2008). Se analizaron 108 artículos, obtenidos de las bases de datos bibliográficas Scopus y Web of Science durante el periodo 2013 a 2017. Se presentan datos sobre autores, revistas, líneas de investigación y los principales resultados, a manera de

\footnotetext{
Tecnológico de Monterrey, México

vcantu7@itesm.mx glasserman@itesm.mx solramirez@ itesm.mx

INVESTIGACIÓN BIBLIOTECOLÓGICA, vol.33, núm. 79, abril/junio, 2019, México, ISSN: 2448-8321 pp. 99-117
} 
indicadores que se tomaron en cuenta al analizar los estudios. Se encontró que existe un número limitado de artículos cuyo objeto de estudio es la evaluación de la educación en emprendimiento dentro de un contexto académico. La mayoría de ellos se han publicado en revistas británicas de acceso cerrado. En cuanto a los autores, son pocos los especialistas en el tema y la mayoría están en Europa Occidental y Norteamérica. Por último, las líneas de investigación resultaron ser muy variadas, así como los principales resultados; la mayoría de los estudios se centra en niveles de educación superior del área de negocios.

Palabras clave: Emprendimiento; Evaluación; Educación; Métricas

\section{Metric behavior on evaluation of educaction in entre- preneurship \\ Valeria Cantú González, Leonardo David Glasserman \\ Morales and María Soledad Ramírez Montoya}

\section{Abstract}

This article aimed to show the results of a systematic map on the evaluation of entrepreneurship education. The objective was to identify the most recent publications on the subject, which were published in journals indexed in Scopus and Web of Science databases. A systematic mapping was developed following the methodology of Petersen et al. (2008). We analyzed 108 articles found in Scopus and Web of Science databases from 2013 to 2017. Authors, journals, research lines and main results were some of the indicators that were taken into account in analyzing the studies. It was found that there are few articles that study the evaluation of entrepreneurship education, within an academic context. Most of them have been published in British journals of closed-access. As for the authors, there are few specialists in the subject who have published more than one article on the subject. Most of these authors are from Western Europe and North America. Finally, the research lines turned out to be quite varied; as well as the main findings. Most of the studies focused on higher education levels in the business area.

Keywords: Entrepreneurship; Evaluation; Assessment; Education; Metrics 


\section{INTRODUCCIÓN}

— $\mathrm{n}$ los últimos años, el sector empresarial ha implementado cambios aceElerados, lo cual implica adecuaciones en su manera de proceder con el fin de mantener una industria competitiva. De acuerdo con Fayolle (2007a), se ha tenido que pensar en formas creativas de incorporar innovaciones e idear nuevas maneras de diseñar y reestructurar a la empresa con el fin de mantener o alcanzar el éxito en su área. De tal manera, una de las estrategias que se han puesto en marcha es empezar a educar en el área de emprendimiento a los futuros empresarios (Fayolle, 2007b; OECD, 2009).

La palabra emprendimiento nace del vocablo francés entrepreneur, el cual se refiere a iniciar algo y estar listo para la toma de decisiones (Rodríguez, 2009). El término emprendimiento no se ha mantenido estático. Entre los siglos XVII y XVIII se catalogaba a los arquitectos como personas emprendedoras, pues creaban edificios y obras nuevas; fue así como se relacionó el término emprendimiento con las empresas, pues éstas involucraban aspectos económicos y creaban productos nuevos, mientras se tomaban decisiones para llegar a los objetivos deseados. Fue hasta 1755, gracias a Richard Cantillón, en Francia, que se introduce el término moderno de entrepreneur, que designaba a una persona que tomaba riesgos en condiciones variables y de incertidumbre, y que a su vez estaba a cargo de trabajadores que recibían salarios, mientras que el emprendedor obtenía las ganancias de la empresa (Formichella, 2004; Rodríguez, 2009). La característica principal de un emprendedor es su actitud, ya que no sólo ve su alrededor, sino que lo observa y percibe cualquier oportunidad que pueda surgir para generar nuevas ideas y nuevos proyectos (Formichella, 2004).

Hoy en día se puede definir al emprendimiento como una manera de razonar, pensar y actuar, donde las oportunidades, las aproximaciones holísticas y el liderazgo balanceado es primordial. Por su parte, un emprendedor es aquella persona capaz de catalizar cambios y crear nuevas empresas (Abu-Saifan, 2012). La mayor aplicación de dichos términos ha sido en el ámbito de negocios, de tal modo que la mayoría de las investigaciones se han concentrado en este rubro (Aldana, Ibarra y Loewenstein, 2011; Cancino, Coronado y Farías, 2012; Duarte, 2007; Sepúlveda, 2016). No obstante, el emprendimiento se ha incorporado a distintas áreas además de la empresarial, como en el caso del campo educativo.

Personas de distintos rubros, como el económico, social y pedagógico, han expresado su preocupación por la relación que existe entre la educación y el empleo (Bas Peña, 2007; Martínez-Mediano, Lord y Rioperez Losada, 2013); es en el emprendimiento donde la educación juega un papel de suma 
importancia, desde la transmisión de contenidos y habilidades hasta las actitudes necesarias para ser un buen emprendedor (OECD, 2009). El papel de la educación, en relación con el emprendimiento, es cada vez mayor debido a los constantes cambios de la economía global; es de suma importancia formar a los estudiantes para que sean capaces de adaptarse a dichos cambios y su vez generar nuevas ideas para resolver los problemas que se viven en el mundo (Luis, Palmero, y Escolar, 2015).

La OECD (2009) señala que la educación para programas sobre emprendimiento se refiere a la transferencia de "habilidades y atributos, incluyendo la habilidad de pensar creativamente, de trabajar en equipo, de manejar los riesgos y las incertidumbres" (OECD, 2009: 5). Los programas sobre emprendimiento pueden ser impartidos en múltiples niveles educativos, es decir, no son exclusivos para nivel superior o posgrado; al contrario, se recomienda comenzar desde edades tempranas con el fin de ir fomentando las habilidades y actitudes necesarias para ser emprendedores (OECD, 2009).

Generalmente, la formación en emprendimiento suele ofrecerse en tres versiones: 1) programas basados en la educación, 2) esquema de premios, y 3) esquema de vinculación. La primera opción se refiere a los que las instituciones ofrecen actualmente. La mayoría han estado orientados a nivel bachillerato y universitario, donde los cursos y programas están enfocados hacia el emprendimiento. En relación con el esquema de premios, se trata de concursos que tienen por objetivo fomentar el emprendimiento a través de la creación de pequeñas empresas y generación de ideas innovadoras. Por último, el esquema de vinculación se refiere a los convenios que se generan entre la academia y la industria, de tal forma que los estudiantes tengan la oportunidad de vivir un ambiente real de trabajo. En este tipo de proyectos se les solicita que generen ideas, proyectos o prototipos y son guiados por mentores del sector empresarial (OECD, 2009).

Ya sea que se trate de un curso o de un programa de emprendimiento, deberá incluir dentro de su planeación la enseñanza de actitudes relativas al buen emprendedor. Rasheed (2000) enlista algunas actitudes que favorecen la acción emprendedora: necesidad de logro, autoestima, autoconfianza y autoeficacia.

En el mismo sentido que las actitudes, se encuentra el espíritu emprendedor. Este espíritu está estrechamente ligado a la socialización. Existen dos tipos de socialización: la primera ocurre al inicio de la vida de una persona, con su familia y allegados, la secundaria cuando ingresa al sistema educativo y comienza a convivir con personas de otros círculos. Esta socialización es muy importante puesto que, si el individuo no estuvo en contacto con un ambiente y personas emprendedoras, será más difícil que lo sea (Formichella, 2004). De este modo, se confirma el papel crucial que juega la educación; si 
la escuela proporciona ambientes donde se fomente el emprendimiento, estará forjando el espíritu emprendedor en sus estudiantes.

Ahora bien, en cuanto a la evaluación del emprendimiento, la OECD publicó un documento en 2007 donde se daba a conocer un marco de referencia para evaluar pequeñas y medianas empresas, así como las políticas y programas sobre emprendimiento. En el documento se estableció que la evaluación de programas sobre emprendimiento debe ser parte integral de las políticas establecidas. Se recomienda realizar evaluaciones formativas mientras la política nueva es puesta en marcha, y una evaluación sumativa una vez que se puedan medir los efectos. Este último tipo de evaluación se debe realizar de manera tanto cuantitativa, utilizando métodos estadísticos, como cualitativa, con el estudio de casos o revisión de pares (OECD, 2007).

En resumen, el tema del emprendimiento va tomando mayor fuerza; sin embargo, presenta áreas de oportunidad, tal es el caso de la evaluación. Aunque existe literatura que habla sobre políticas y lineamientos para evaluar programas de amplio rango, hace falta información acerca de cómo debe ser la evaluación de un curso o programa universitario de emprendimiento. Algunos estudios bibliométricos, como el de Kakouris y Georgiadis (2016), confirman la escasez cuantitativa en temas de investigación en el campo del emprendimiento. Asimismo, Landström, Harirchi y Åström (2012) mencionan que al parecer el emprendimiento es un área muy cercana a los estudios de gestión y la economía, pero que se esperaría una mayor integración entre el campo del emprendimiento y otras áreas en el futuro. Destaca el hecho de que es a partir del 2000 cuando el emprendimiento se incluye como un área educativa en la base de datos Web of Science (WoS). En tanto, Loi, Castriotta y Di Guardo (2016) mencionan que recientemente ha surgido interés en temas de evaluación y aprendizaje en el ámbito empresarial, donde se incluye experimentación continua con métodos, enfoques de enseñanza y marcos de evaluación para generar nuevos conocimientos y aportes teóricos.

Si bien existen estudios relacionados con el tema, no se observa una línea específica que aborde la evaluación de la enseñanza del emprendimiento. Es ahí donde resalta la importancia de la bibliometría en el análisis de dominios científicos y la revisión bibliográfica de trabajos bibliométricos sobre emprendimiento, ya que permiten identificar cuál es el aporte del presente estudio, el cual se enfoca en dar a conocer qué es lo que se ha escrito sobre la evaluación de la educación para el emprendimiento. Se desea conocer quién escribe, en dónde lo hace, en qué se enfoca su investigación, entre otros aspectos, con miras a ubicar el potencial del tema y abordar al diseño de un programa de posgrado de emprendimiento situado en el área de humanidades (educación). 


\section{Metodología}

Los mapeos sistemáticos se incluyen en distintas áreas de conocimiento. Petersen et al. (2008) proponen un método para llevar a cabo mapeos sistemáticos adaptados al campo de las ingenierías. Esta adaptación se basa en la metodología propuesta por Kitchenham (2007) para la realización de revisiones sistemáticas de literatura. La presente investigación siguió el método propuesto por Petersen et al. (2008), el cual consta de cinco pasos claves: 1) definición de las preguntas de investigación, 2) realización de la búsqueda, 3) selección de trabajos, 4) creación de palabras clave usando los resúmenes, y 5) extracción de datos y creación de mapa. A continuación se describe el proceso que se llevó a cabo.

\section{Definición de las preguntas de investigación}

Esta etapa consiste en definir con claridad qué es lo que se pretende saber al hacer el mapeo. El propósito principal de este estudio fue conocer qué se ha documentado e investigado en relación con la evaluación de la educación en emprendimiento. Con base en dicho objetivo, las preguntas que se pretendieron resolver son las siguientes:

RQ1 ¿Cuántos artículos científicos se tienen con respecto al tema de evaluación de la educación en emprendimiento entre 2013 y 2017?

RQ2 ¿Cuál es la afiliación institucional de los autores que han publicado sobre el tema?

RQ3 ¿Cuáles son las revistas en las que se han publicado artículos al respecto y en qué países tienen su adscripción?

RQ4 ¿Cuáles son las líneas de investigación que se evidencian sobre el tema?

\section{Realización de la búsqueda}

Esta etapa consistió en la identificación de las bases de datos y la búsqueda de libros, artículos, conferencias y tesis relacionadas con la temática. Se revisaron las bases de datos Scopus y Web of Science (WoS) por ser dos de las principales bases de datos bibliográficas multidisciplinarias. En ambas fuentes se realizó una búsqueda de artículos relacionados con las siguientes combinaciones 
de palabras en inglés: entrepreneurship AND assessment, entrepreneurship AND evaluation. La búsqueda se realizó con palabras en inglés debido a que ambas bases de datos son administradas en ese idioma. Por otro lado, no se utilizó el truncamiento *, pues el objeto de estudio fue muy específico. Los pasos que se siguieron para realizar la búsqueda en ambas bases de datos fueron los siguientes: primero, se introdujo el primer par de palabras mencionadas líneas arriba, limitando la búsqueda de 2013 a julio de 2017; después, una vez arrojados los primeros resultados, se utilizaron filtros como idioma (inglés y español) y tipo de documento (artículos), además de categoría. No obstante, debido a que las dos bases de datos utilizadas no presentan la misma clasificación de categorías, la búsqueda resultó de la siguiente manera: en Scopus se filtraron los resultados con base en las categorías de a) Ciencias Sociales y b) Psicología; mientras que en WoS se tomaron en consideración los artículos pertenecientes a las categorías a)Educación, investigación educativa, b) Educación, disciplinas científicas, c) Ciencias Sociales interdisciplinarias, d) Psicología multidisciplinar, e) Psicología, y f) Psicología educativa.

\section{Selección de trabajos}

En esta etapa es importante documentar la manera en la que se seleccionan los documentos de interés y asegurar que sea replicable en búsquedas futuras. De acuerdo con Petersen et al. (2008), se deben especificar ciertos criterios de inclusión y exclusión de tal modo que sirvan de un segundo filtro y así depurar la base de trabajos encontrados. Este paso, como lo indica el autor, se realiza a través de la lectura de los resúmenes de los artículos.

Para el presente caso, se incluyeron aquellos artículos que tuvieran las palabras entrepreneurship o entrepreneurial en el título o resumen. Además, se tomaron en cuenta las investigaciones realizadas en el campo académico dentro de alguna institución educativa. En otras palabras, se incluyeron investigaciones referentes a planes curriculares, clases de emprendimiento o programas desarrollados dentro de una institución académica. En el caso opuesto, se dejaron fuera aquellos artículos que presentaran los términos social entrepreneurship o social entrepreneurial en el título o en el resumen. También se excluyeron los estudios que se realizaron en otros contextos fuera del académico, tal es el caso de las investigaciones en empresas o en la política. 


\section{Creación de categorías clave usando los resúmenes}

El método propuesto por Petersen et al. (2008) señala que una vez depurada la base de datos de los trabajos encontrados, se lean únicamente los resúmenes de éstos. Durante la lectura se identifican las categorías clave que puedan ser usadas para agrupar los estudios de la base de datos. En el caso de los artículos relacionados con la evaluación de la educación en emprendimiento, se leyeron los resúmenes con tres objetivos en mente: 1) qué tipo de investigación se aborda, 2) cuál es la temática de investigación, y 3) cuál es el resultado del estudio realizado. En relación con el primer objetivo, el tipo de investigación, se utilizó la clasificación de Kumar (2008), en la cual se menciona que los trabajos de investigación pueden ser empíricos o conceptuales. Los primeros son estudios basados en la experiencia u observaciones, mientras que los conceptuales aluden a los trabajos enfocados a estudiar aspectos abstractos o teoría, como revisiones de literatura. Por otro lado, en cuanto a las líneas de investigación y resultados, las categorías emergieron de la información que se extrajo de los resúmenes debido a que no existe literatura que enuncie las distintas temáticas que se pueden evaluar sobre la educación en emprendimiento.

\section{Extracción de datos y creación de mapa}

Por último, es importante completar todos los metadatos en el archivo de registro de artículos identificados (base de datos), incluyendo las categorías que den respuesta a las preguntas de investigación. Posteriormente, se continúa con el análisis de la información.

Para el presente artículo se generó la base de datos que se puede consultar en https://tinyurl.com/yab8ygxt. El análisis se realizó con el software Microsoft Excel.

\section{Presentación y anÁlisis de Resultados}

De los 266 artículos que arrojó la búsqueda inicial (resultados de Scopus y WoS, se eliminaron los artículos repetidos), después de haber seguido la metodología, resultó una base de 108 estudios publicados en revistas científicas durante el periodo que comprende de enero de 2013 a julio de 2017. En la Tabla 1 se muestra que cerca de $88 \%$ de los trabajos fueron recuperados de la base de datos Scopus. En WoS se encontraron 41 artículos, no obstante, 30 de ellos también se localizaron en Scopus. En cuanto al tipo de artículo, $76.8 \%$ corresponde a estudios empíricos y el resto a conceptuales o teóricos. 


\begin{tabular}{|l|c|c|c|}
\hline \multirow{2}{*}{ Base de datos } & \multicolumn{2}{|c|}{ Tipo de artículo } & \multirow{2}{*}{ Total } \\
\cline { 2 - 3 } & Conceptual & Empírico & 67 \\
\hline Scopus & 18 & 49 & 11 \\
\hline WoS & 2 & 9 & 30 \\
\hline WoSy Scopus & 5 & 25 & 108 \\
\hline Total & 25 & 83 & 0 \\
\hline
\end{tabular}

Tabla 1. Tipo de artículos por base de datos

La evolución de la publicación de artículos, tanto empíricos como conceptuales, sobre evaluación de la educación en emprendimiento ha ido en aumento, como lo muestra la Figura 1. Se observa que el tema es ascendente a pesar de haber tenido un ligero retroceso en 2016. Es preciso puntualizar que una limitante del estudio es que la investigación terminó en julio de 2017, es por ello que dicho año reporta una baja de producción, por lo que pueden existir documentos posteriores a esa fecha que no se consideraron.

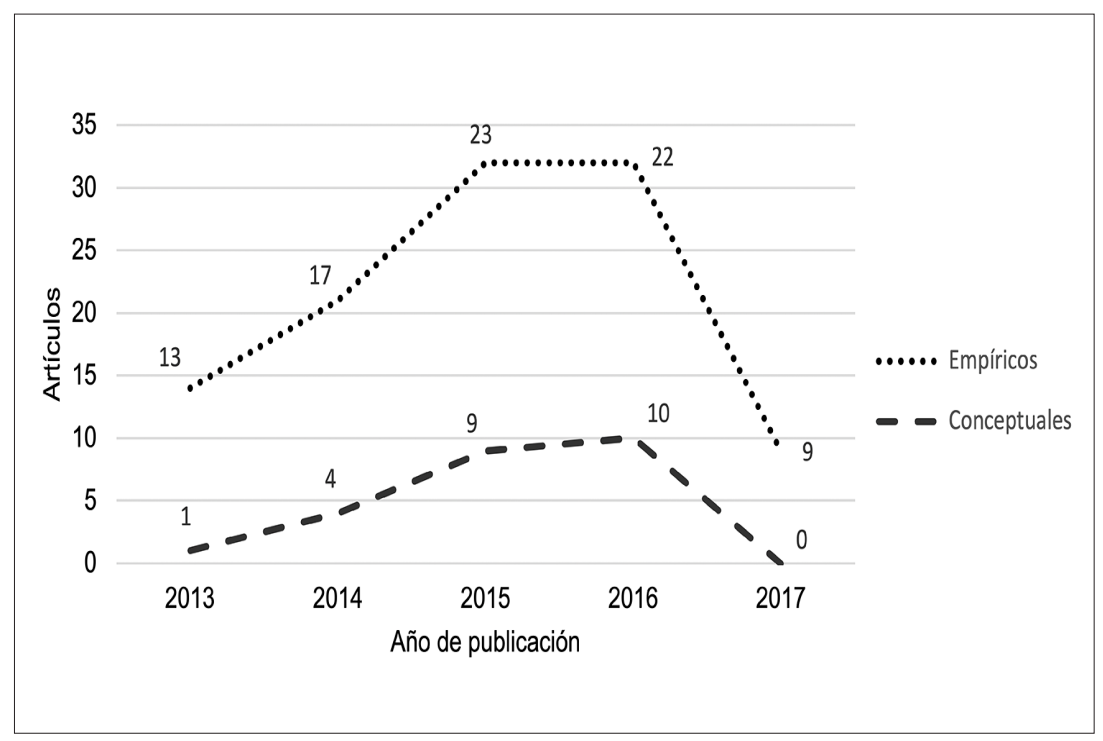

Figura 1. Tipo de artículos publicados anualmente

En relación con los autores de los artículos, la Tabla 2 enlista aquellos autores que registraron más de una publicación en el periodo de tiempo en el cual se delimitó la búsqueda de artículos. Para realizar dicha lista, se tomaron en cuenta tanto autores principales como coautores. 


\begin{tabular}{|l|l|}
\hline \multicolumn{1}{|c|}{ Autor } & n \\
\hline Pedrosa, I. & 4 \\
\hline Suárez-Álvarez, J. & 4 \\
\hline García-Cueto, E. & 3 \\
\hline Maritz, A. & 3 \\
\hline Muñiz, J. & 3 \\
\hline Bernal Guerrero, A. & 2 \\
\hline Galiana, L. & 2 \\
\hline Moberg, K. & 2 \\
\hline Oliver, A. & 2 \\
\hline Shartrand, A. & 2 \\
\hline
\end{tabular}

Tabla 2. Producción de autores entre 2013 y 2017

Por otro lado, en la Figura 2 se muestran las regiones a las cuales pertenecen los autores de los 108 estudios que contempla la base de datos, con base en la afiliación institucional del primer autor. Europa Occidental es la principal zona que escribe sobre el tema, seguida de Norteamérica y Asia. Un dato que sobresale es que Centroamérica no tiene producción alguna en el periodo que comprende la revisión (2013-2017). Los principales países de cada región que han escrito sobre el tema son España, Estados Unidos de américa, China, Malasia, Croacia y República Checa, Sudáfrica, Australia y Colombia.

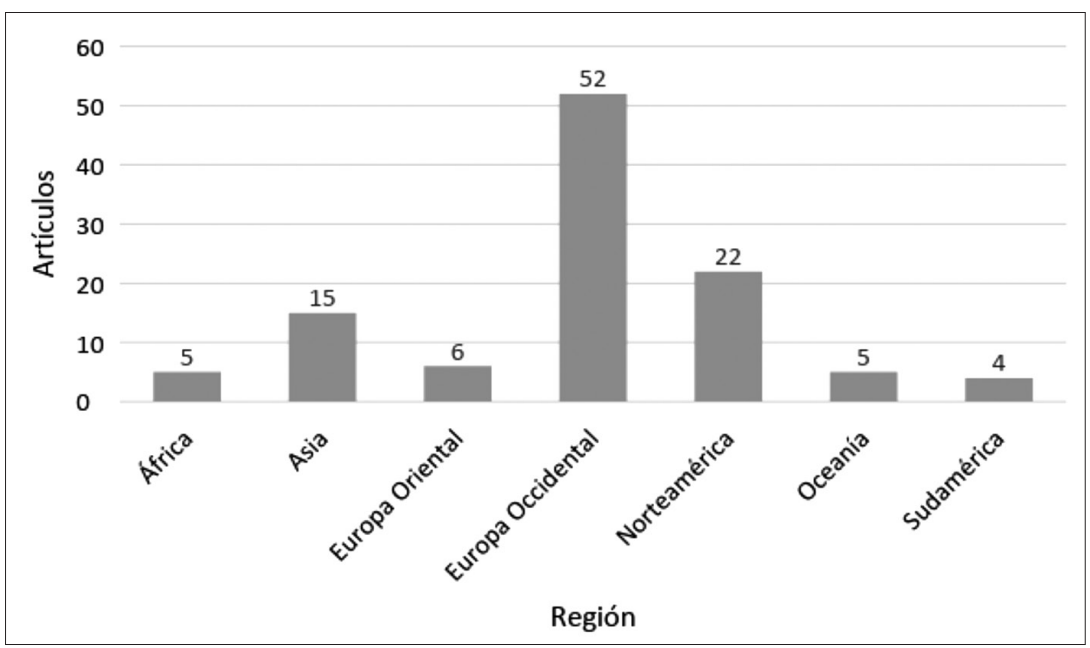

Figura 2. Producción de artículos por regiones según adscripción de autores Nota: La clasificación de las regiones es elaboración propia de los autores 
En lo que respecta a las revistas que han publicado sobre el tema, se muestra en el Anexo el listado que incluye país de procedencia y el indicador de impacto basado en el SCImago Journal Rank (SJR, por sus siglas en inglés). Europa Occidental resultó con la mayor cantidad de artículos publicados, esto es, $71.3 \%$ de la producción total, seguido de Norteamérica con $15.7 \%$. Sudamérica, Europa del Este y Asia publicaron en sus revistas 3.7 \% cada región. Por último, Oceanía y África sólo obtuvieron 0.9 \% de la producción total cada una de las regiones.

Gran Bretaña es el país que más artículos publicó. Sobresalen dos revistas: Education and Training (Q2) y Journal of Entrepreneurship Education (Q3) con 16 y 10 artículos publicados respectivamente. Por otro lado, con respecto al tipo de acceso, solamente 12 de las 66 revistas encontradas son de acceso abierto.

Después de analizar descriptivamente la base de datos, se procedió a leer los resúmenes de cada estudio con el fin de identificar cuál era su enfoque de investigación. Si bien en total fueron 108 artículos los que se identificaron, en ocasiones una misma investigación tuvo por objeto de estudio dos líneas, de ahí que el conteo sea mayor a dicho número (Tabla 3).

En lo que respecta a los trabajos de corte empírico, codificados en la base de datos con la letra E, la mayoría de los estudios se enfoca en evaluar programas ya existentes sobre emprendimiento (31 artículos) o bien tienen por objeto de estudio identificar cuáles son las principales actitudes para ser un emprendedor (15 artículos). En relación con el primer tema, el objetivo de los artículos fue presentar evidencia sobre el funcionamiento positivo o negativo de un programa, o bien conocer su impacto. En lo que se refiere a las actitudes, algunos estudios hicieron uso de instrumentos ya existentes que tienen como propósito medir actitudes en general o actitudes necesarias para el emprendimiento. Otros estudios, por su parte, presentaban un corte cualitativo y se enfocaron en preguntar acerca de las actitudes principales para un emprendedor. El resto de las líneas se centraba en diversos temas: instrumentos, creación, implementación o validación de instrumentos; percepción sobre emprendimiento, sobre los distintos agentes de la educación en el emprendimiento; habilidades de emprendimiento, evaluación de las habilidades en torno al emprendimiento; implementación de programas, creación e implementación de programas sobre emprendimiento; espíritu emprendedor, evaluación del espíritu emprendedor; otras líneas de artículos empíricos, diferentes temas que no se ajustaban al resto de las categorías.

En cuanto a los estudios conceptuales o teóricos, la línea que punteó fue educación en emprendimiento (13 artículos). En sí, son estudios que tuvieron como fin revisar literatura sobre el tema y tratar de esclarecer ciertos 
conceptos. Las otras líneas, educación en emprendimiento y aspectos pedagógicos, hacen mención de aspectos específicos como estrategias de enseñanza-aprendizaje, actitudes, habilidades, entre otras; otras líneas de artículos conceptuales trata temas que no se ajustan al resto de las líneas.

\begin{tabular}{|c|c|c|c|}
\hline Tipo de artículo & Línea & Código de artículo & $\mathrm{n}$ \\
\hline \multirow[t]{8}{*}{ Empírico } & $\begin{array}{l}\text { Programas } \\
\text { de emprendimiento }\end{array}$ & $\begin{array}{l}\text { E4, E5, E11, E13, E15, E16, E19, E21, } \\
\text { E24, E28, E32, E33, E34, E39, E40, } \\
\text { E43, E45, E46, E51, E53, E56, E58, } \\
\text { E59, E62, E63, E70, E72, E77, E79, } \\
\text { E80, E81 }\end{array}$ & 31 \\
\hline & Instrumentos & $\begin{array}{l}\text { E10, E35, E62, E66, E67, E73, E74, } \\
\text { E76 }\end{array}$ & 8 \\
\hline & Actitudes de emprendimiento & $\begin{array}{l}\text { E1, E8, E22, E26, E36, E42, E44, } \\
\text { E49, E52, E57, E68, E71, E73, E74, } \\
\text { E75 }\end{array}$ & 15 \\
\hline & $\begin{array}{l}\text { Percepción sobre empren- } \\
\text { dimiento }\end{array}$ & $\begin{array}{l}\text { E3, E4, E9, E17, E18, E37, E38, E47, } \\
\text { E53, E61, E69 }\end{array}$ & 11 \\
\hline & $\begin{array}{l}\text { Habilidades sobre } \\
\text { emprendimiento }\end{array}$ & E28, E33, E37, E65 & 4 \\
\hline & $\begin{array}{l}\text { Implementación } \\
\text { de programa }\end{array}$ & $\begin{array}{l}\text { E2, E6, E7, E14, E23, E31, E54, E55, } \\
\text { E60, E78, E82, E84, E85 }\end{array}$ & 13 \\
\hline & Espíritu emprendedor & E21, E38, E46, E50, E81 & 5 \\
\hline & $\begin{array}{l}\text { Otras líneas de artículos } \\
\text { empíricos }\end{array}$ & $\begin{array}{l}\text { E12, E20, E25, E27, E29, E30, E41, } \\
\text { E48, E64 }\end{array}$ & 9 \\
\hline \multirow[t]{3}{*}{ Conceptual } & $\begin{array}{l}\text { Educación } \\
\text { en emprendimiento }\end{array}$ & $\begin{array}{l}\mathrm{C} 1, \mathrm{C} 5, \mathrm{C} 6, \mathrm{C} 7, \mathrm{C} 8, \mathrm{C} 10, \mathrm{C} 12, \mathrm{C} 13, \\
\mathrm{C} 14, \mathrm{C} 16, \mathrm{C} 18, \mathrm{C} 21, \mathrm{C} 23\end{array}$ & 13 \\
\hline & $\begin{array}{l}\text { Educación } \\
\text { en emprendimiento } \\
\text { y aspectos pedagógicos }\end{array}$ & C3, C9, C17, C19, C20 & 5 \\
\hline & Otros líneas conceptuales & C2, C4, C11, C15, C22, C24 & 6 \\
\hline
\end{tabular}

Tabla 3. Principales líneas de investigación

\section{Discusión}

Después de haber realizado un mapeo sistemático sobre el tema delimitado entre el periodo de enero de 2013 a junio de 2017, resultó que existen 108 artículos en las bases de datos Scopus y WoS que abordan el tema, además de ser estudios realizados en o sobre un contexto académico. Es evidente que la mayoría son investigaciones empíricas en las cuales se evaluó, aplicó o cuestionó algún aspecto en relación al tema, y la gran mayoría fueron realizados 
en el área de negocios (Aldana, Ibarra y Loewenstein, 2011; Cancino, Coronado y Farías, 2012; Duarte, 2007; Sepúlveda, 2016). Asimismo, se encontró una mayor cantidad de artículos en Scopus en relación con los obtenidos en WoS. Lo anterior se puede atribuir a los criterios de inclusión de revistas en cada una de las bases de datos bibliográficas estudiadas.

Son pocos los autores que se han especializado en el tema de la evaluación de la educación para el emprendimiento. De los 291 autores que publicaron artículos, ya sea en coautoría o en individual, solamente 10 de ellos publicaron más de un estudio. Dos autores (Pedrosa y Suárez-Álvarez), con afiliación a una institución española, obtuvieron el mayor número de artículos, los cuales ascienden a cuatro en total. En cuanto a la adscripción de los autores, la mayoría pertenecía a instituciones de España y Gran Bretaña. Es preciso señalar que existe una considerable desproporción en la distribución de los autores en el mundo. Son pocos los investigadores de África, Europa del Este, Oceanía y Sudamérica que han publicado acerca del tema; sin embargo, resalta más aún el hecho de que no existen autores de Centroamérica con publicaciones en la temática en revistas indexadas a Scopus o WoS. Asimismo, es preciso señalar que al parecer no existe un referente de peso en el tema en cuestión.

Existe una gran variedad de revistas que publican temas relacionados con evaluación de la educación para el emprendimiento, no obstante, muy pocas son de acceso abierto. Los artículos se distribuyeron en un total de 71 revistas. La mayoría de las publicaciones fueron realizadas en revistas de origen británico, estadounidense o español, de acceso cerrado. Lo anterior limita que cualquier persona pueda tener acceso a ese tipo de información y así implementar de una mejor manera la evaluación sobre la educación en emprendimiento.

Una gran cantidad de estudios se han centrado en evaluar programas de emprendimiento. Al analizar las diferentes temáticas o líneas de investigación, se lograron identificar ocho líneas de estudios empíricos y tres de conceptuales. La gran mayoría de los artículos de corte empírico tuvieron como objetivo de investigación evaluar programas de emprendimiento (de los tres tipos que establece la OECD (2009): programas basados en la educación, esquema de premios y esquema de vinculación), implementar programas de emprendimiento, evaluar las actitudes relacionadas al emprendimiento y evaluar las percepciones de distintos agentes sobre el emprendimiento. Si se analizan estas temáticas con las planteadas por la OECD (2007), se observa que no existen coincidencias. Los lineamientos establecidos por la OECD están más enfocados en cuestiones generales, como el tipo de enfoque que se recomienda, ya sea cualitativo o cuantitativo. No obstante, lo que se logró 
identificar en el presente mapeo es que hay muchas líneas de investigación, según las necesidades de cada caso.

Por lo tanto, se concluye que el aporte que ofrece el presente estudio bibliométrico está en función de la identificación de autores, revistas, líneas de investigación y principales resultados de investigaciones que aborden la temática de evaluación de la enseñanza del emprendimiento; destaca que no existen suficientes estudios en educación formal y distinta de las áreas de negocios y economía.

\section{CONSIDERACIONES FINALES}

En resumen, hacen falta más estudios sobre la evaluación de la educación para el emprendimiento, de acceso abierto, de tal modo que se pueda llegar a utilizar una misma metodología que fomente el desarrollo de futuros emprendedores. Otra área de oportunidad son las investigaciones conceptuales, pues existe una ausencia de estudios que den la pauta para fundamentar la evaluación de este tipo. La mayoría de los artículos abordan la investigación en implementación o evaluación de algún aspecto en particular; sin embargo, falta literatura que sustente dicho campo. En cuanto a las áreas en las cuales se han realizado los estudios, aunque no se especifica en el presente artículo, la gran mayoría son en el área de negocios; será importante voltear a ver otras disciplinas tal y como lo indican Landström, Harirchi y Åström (2012). Se sugiere ampliar el campo de estudio en el cual se pueda innovar y vincular los estudios y programas formativos con posibilidad de hacerlo. Como lo indica la OECD (2009), la educación para el emprendimiento se puede dar desde el nivel básico hasta el posgrado. Futuras líneas de investigación podrían concentrar los esfuerzos en documentar la metodología para enseñar el emprendimiento y no limitarse a la educación en emprendimiento en negocios, sino ampliar el alcance a otras disciplinas como las humanidades, pedagogía, industrias creativas, por mencionar algunos. Al girar el enfoque de estudio, se podría generar conocimiento susceptible de ser transferido a diversos sectores (públicos, privados, gubernamentales, organizaciones no gubernamentales), que aporte valor a la sociedad, permita soluciones a las problemáticas locales y globales y que se generen nuevas oportunidades de mejora y crecimiento económico y social. Es importante fomentar en las nuevas generaciones las habilidades y actitudes relacionadas al emprendimiento, y qué mejor que poniendo el ejemplo desde el propio campo educativo. 
Esta investigación es producto del Proyecto 266632 "Laboratorio Binacional para la Gestión Inteligente de la Sustentabilidad Energética y la Formación Tecnológica”, financiado por el fondo CONACYT SENER para la Sustentabilidad Energética (Acuerdo: S0019201401).

\section{REFERENCIAS}

Abu-Saifan, Samer. 2012. "Social entrepreneurship: definition and boundaries". Technology Innovation Management Review 2 (2). http://timreview.ca/article/523

Aldana, Eugenia del Carmen, María Teresa Ibarra e Ingrid Loewenstein. 2011. "El modelo de negocios como reforzador del emprendimiento en las universidades. Caso del Tecnológico de Monterrey campus ciudad de México”. Revista Ciencias Estratégicas 19 (26): 185-201. http://www.redalyc.org/articulo.oa?id=151322415004

Bas Peña, Encarna. 2007. "El Prácticum en la Titulación de Pedagogía: discurso y práctica profesional”. Pedagogía Social. Revista Interuniversitaria 14: 139-153. doi:10.71797/PSRI.

Cancino, Christian, Freddy Coronado y Antonio Farías. 2012. "Antecedentes y resultados de emprendimientos dinámicos en Chile: cinco casos de éxito”. INNOVAR. Revista de Ciencias Administrativas y Sociales 22 (43): 19-32. http://www.redalyc. org/articulo.oa?id=81824123003

Clapton, Janet, Deborah Rutter y Nadira Sharif. 2009. SCIE Systematic mapping guidance. Consultado el 3 de julio, 2017. http://lx.iriss.org.uk/sites/default/files/resources/rr03.pdf

Diccionario de la Lengua Española. 2017. "Emprender”. Fecha de consulta: 23 de junio de 2017. http://dle.rae.es/?id=Esip2Nv

Duarte, Franklin. 2007. "Emprendimiento, empresa y crecimiento empresarial”. Contabilidady Negocios 2 (3): 46-56. http://www.redalyc.org/articulo.oa?id=281621764007

Fayolle, A. 2007a. Handbook of research in entrepreneurship education, volume 1. A general perspective. Gran Bretaña: Edward Elgar.

Fayolle, A. 2007b. Handbook of research in entrepreneurship education, volume 2. Contextual Perspectives. Gran Bretaña: Edward Elgar.

Formichella, María Marta. 2004. El concepto de emprendimiento y su relación con la educación, el empleo y el desarrollo local. Buenos Aires, Argentina. Fecha de consulta: 18 de julio de 2017. http://municipios.unq.edu.ar/modules/mislibros/archivos/MonografiaVersionFinal.pdf

Kakouris, Alexandros y Panagiotis Georgiadis. 2016. "Analysing entrepreneurship education: a bibliometric survey pattern". Journal of Global Entrepreneurship Research 6 (6). doi: 10.1186/s40497-016-0046-y

Kitchenham, Barbara. 2007. Guidelines for performing systematic literature reviews in software engineering. Technical Report EBSE-2007-01. School of Computer Science and Mathematics, Keele University. Fecha de consulta: 27 de junio de 2017. https:// pdfs.semanticscholar.org/e62d/bbbbe70cabcde3335765009e94ed2b9883d5.pdf 
Kumar, Ranjit. 2008. Research methodology. Nueva Delhi: APH Publishing Corporation. Landström, Hans, Gouya Harirchi y Fredrik Åström. 2012. "Entrepreneurship: Exploring the knowledge base”. Research Policy 41 (7): 1154-1181. doi: 10.1016/j.respol.2012.03.009

Loi, Michela, Manuel Castriotta y Maria Chiara Di Guardo. 2016. "The theoretical foundations of entrepreneurship education: How co-citations are shaping the field”. International Small Business Journal: Researching Entrepreneurship 34 (7): 948-971. doi:10.1177\%2F0266242615602322

Luis, Ma. Isabel, Carmen Palmero y María Camino Escolar. 2015. "Impacto de la educación en el emprendimiento. Making-of y análisis de tres grupos de discusión”. Pedagogía Social. Revista Interuniversitaria, 25, 221-250. http://www.redalyc.org/articulo.oa?id=135043709013

Martínez-Mediano, Catalina, Susan Lord y Nuria Rioperez Losada. 2013. "Programa de Desarrollo de Competencias para el Aprendizaje a lo Largo de la Vida para Estudiantes de Educación Superior". Pedagogía Social. Revista Interuniversitaria, 22, 137-151. doi:10.7179/PSRI.

Petersen, Kai, Robert Feldt, Shahid Mujtaba y Michael Mattsson. 2008. "Systematic Mapping Studies in Software Engineering”. EASE, 8: 68-77. http://www.robertfeldt.net/publications/petersen_ease08_sysmap_studies_in_se.pdf

Rasheed, Howard. 2000. "Developing entrepreneurial potential in youth of entrepreneurial education and venture creation”. University of North Carolina at Wilmintong. Fecha de consulta: 15 de julio de 2017. http://www.usasbe.org/knowledge/proceedings/2001/063.pdf

Rodríguez, Alfonso. 2009. "Nuevas perspectivas para entender el emprendimiento empresarial”. Pensamiento E Gestión, 26: 94-119. http://www.redalyc.org/ pdf/646/64612291005.pdf

Sepúlveda, Claudia. 2016. "Sostenibilidad de los emprendimientos: Un análisis de los factores determinantes”. Revista Venezolana de Gerencia 21 (73): 33-49. http://www.redalyc.org/pdf/290/29045347003.pdf

OECD (The Organisation for Economic Co-operation and Development). 2007. "OECD framework for the evaluation of SME and entrepreneurship policies and programmes”. OECD. Fecha de consulta: 7 de agosto de 2017. http://www.untag-smd.ac.id/files/Perpustakaan_Digital_1/ENTREPRENEURSHIP\%20 OECD \%20Framework\%20for\%20the\%20 evaluation\%20of \%20SME\%20 and $\% 20$ entrepreneurship $\% 20$ policies $\% 20$ and $\% 20$ progr.pdf

OECD. 2009. "Evaluation of programmes concerning education for entrepreneurship, report by the OECD Working Party on SMEs and Entrepreneurship". Fecha de consulta: 7 de agosto de 2017. https://www.oecd.org/cfe/smes/42890085.pdf 
Anexo

Revistas con mayor número de artículos, SJR y tipo de acceso

\begin{tabular}{|c|c|c|c|c|c|c|c|}
\hline \multirow{2}{*}{ País } & \multirow{2}{*}{ Revista } & \multicolumn{4}{|c|}{ SJR } & \multirow{2}{*}{$\mathrm{S} / \mathrm{R}$} & \multirow[b]{2}{*}{$\mathrm{n}$} \\
\hline & & Q1 & Q2 & Q3 & Q4 & & \\
\hline \multirow{16}{*}{$\begin{array}{l}\text { Gran } \\
\text { Bretaña }\end{array}$} & Education and Training & & 16 & & & & \multirow[t]{16}{*}{44} \\
\hline & $\begin{array}{l}\text { Journal of Entrepreneurship } \\
\text { Education }\end{array}$ & & 10 & & & & \\
\hline & $\begin{array}{l}\text { International Journal of Gende } \\
\text { and Entrepreneurship }\end{array}$ & & 4 & & & & \\
\hline & Spanish Journal of Psychology & & 2 & & & & \\
\hline & $\begin{array}{l}\text { Environment and Planning C: } \\
\text { Government and Policy }\end{array}$ & 1 & & & & & \\
\hline & $\begin{array}{l}\text { European Journal of International } \\
\text { Management }\end{array}$ & & 1 & & & & \\
\hline & $\begin{array}{l}\text { International Journal of Advanced } \\
\text { Media and Communication }\end{array}$ & & 1 & & & & \\
\hline & $\begin{array}{l}\text { Journal of Vocational Education } \\
\text { and Training }\end{array}$ & & 1 & & & & \\
\hline & Teaching and Teacher Education & 1 & & & & & \\
\hline & Studies in Educational Evaluation & 1 & & & & & \\
\hline & Teaching in Higher Education & 1 & & & & & \\
\hline & World Development & 1 & & & & & \\
\hline & World Bank Research Observer & 1 & & & & & \\
\hline & $\begin{array}{l}\text { International Journal of Innovation } \\
\text { and Learning }\end{array}$ & & & 1 & & & \\
\hline & Cultura y Educación & 1 & & & & & \\
\hline & $\begin{array}{l}\text { Journal of Development } \\
\text { Effectiveness }\end{array}$ & & 1 & & & & \\
\hline \multirow[t]{9}{*}{ España } & Psicothema & & 2 & & & & \multirow[t]{9}{*}{11} \\
\hline & Intangible Capital & & & 2 & & & \\
\hline & Anales de Psicología & & 1 & & & & \\
\hline & Educación XX1 & & 1 & & & & \\
\hline & Papeles del Psicólogo & & & 1 & & & \\
\hline & $\begin{array}{l}\text { Ensayos-Revista de la Faculta } \\
\text { de Educación de Albacete }\end{array}$ & & & & & 1 & \\
\hline & Revista de Educación & & 1 & & & & \\
\hline & Espiral-Cuadernos del Profesorado & & & & & 1 & \\
\hline & $\begin{array}{l}\text { Estudios Sobre el Mensaje } \\
\text { Periodístico }\end{array}$ & & 1 & & & & \\
\hline
\end{tabular}




\begin{tabular}{|c|c|c|c|c|c|c|}
\hline \multirow[t]{5}{*}{ Holanda } & $\begin{array}{l}\text { International Journal of Manage- } \\
\text { ment Education }\end{array}$ & & 5 & & & \multirow[t]{5}{*}{9} \\
\hline & $\begin{array}{l}\text { Technological Forecasting } \\
\text { and Social Change }\end{array}$ & 1 & & & & \\
\hline & Social Psychology of Education & 1 & & & & \\
\hline & Higher Education & 1 & & & & \\
\hline & Journal of Affective Disorders & 1 & & & & \\
\hline \multirow[t]{3}{*}{ Italia } & $\begin{array}{l}\text { Mediterranean Journal } \\
\text { of Social Sciences }\end{array}$ & & & 3 & & \multirow[t]{3}{*}{5} \\
\hline & BPA Applied Psychology Bulletin & & & & 1 & \\
\hline & $\begin{array}{l}\text { Journal of Educational, Cultural } \\
\text { and Psychological Studies }\end{array}$ & & & & 1 & \\
\hline \multirow[t]{4}{*}{ Alemania } & $\begin{array}{l}\text { Journal of Teacher } \\
\text { Education for } \\
\text { Sustainability }\end{array}$ & & & 1 & & \multirow[t]{4}{*}{4} \\
\hline & $\begin{array}{l}\text { International Journal of Emerging } \\
\text { Technologies in Learning }\end{array}$ & & & 1 & & \\
\hline & Jahrbuch fur Regionalwissenschaft & & 1 & & & \\
\hline & Vocations and Learning & 1 & & & & \\
\hline Irlanda & $\begin{array}{l}\text { International Journal } \\
\text { of Engineering Education }\end{array}$ & & 3 & & & 3 \\
\hline Suiza & $\begin{array}{l}\text { International Journal } \\
\text { of Management in Education }\end{array}$ & & & 1 & & 1 \\
\hline \multirow{12}{*}{$\begin{array}{l}\text { Estados } \\
\text { Unidos de } \\
\text { América }\end{array}$} & Advances in Engineering Education & & 4 & & & \multirow[t]{12}{*}{15} \\
\hline & $\begin{array}{l}\text { Journal of International Agricultural } \\
\text { and Extension Education }\end{array}$ & & & & 1 & \\
\hline & Hispania & & 1 & & & \\
\hline & Journal of Vocational Behavior & 1 & & & & \\
\hline & $\begin{array}{l}\text { Family and Consumer Sciences } \\
\text { Research Journal }\end{array}$ & 1 & & & & \\
\hline & AIDS Education and Prevention & 1 & & & & \\
\hline & Journal of Career Assessment & & 1 & & & \\
\hline & $\begin{array}{l}\text { American Indian and Alaska Native } \\
\text { Mental Health Research }\end{array}$ & 1 & & & & \\
\hline & $\begin{array}{l}\text { Journal of Professional Issues in } \\
\text { Engineering Education and Practice }\end{array}$ & & 1 & & & \\
\hline & $\begin{array}{l}\text { American Journal } \\
\text { of Pharmaceutical Education }\end{array}$ & 1 & & & & \\
\hline & Advanced Science Letters & & & & 1 & \\
\hline & Chinese Education and Society & & & & 1 & \\
\hline
\end{tabular}




\begin{tabular}{|c|c|c|c|c|c|c|c|}
\hline \multirow[t]{2}{*}{ Canadá } & Innovation Journal & & & 1 & & & 2 \\
\hline & Asian Social Science & & 1 & & & & \\
\hline Polonia & Economics and Sociology & 1 & & & & & 1 \\
\hline Eslovaquia & Studia Psychologica & & & 1 & & & 1 \\
\hline Croacia & Croatian Journal of Education & & & & & 1 & 1 \\
\hline Lituania & Informatics in Education & & 1 & & & & 1 \\
\hline \multirow[t]{2}{*}{ Venezuela } & Revista de Ciencias Sociales & & & 1 & & & 2 \\
\hline & Opción & & & 1 & & & \\
\hline Chile & Formación Universitaria & & & 1 & & & 1 \\
\hline Ecuador & Revista Publicando & & & & & 1 & 1 \\
\hline \multirow[t]{2}{*}{ Turquía } & $\begin{array}{l}\text { Turkish Online } \\
\text { Journal of Educational } \\
\text { Technology }\end{array}$ & & & 2 & & & 3 \\
\hline & $\begin{array}{l}\text { International Journal } \\
\text { of Education in Mathematics } \\
\text { Science and Technology }\end{array}$ & & & & & 1 & \\
\hline China & $\begin{array}{l}\text { Knowledge Management } \\
\text { and E-Learning }\end{array}$ & & 1 & & & & 1 \\
\hline Australia & $\begin{array}{l}\text { Global Journal } \\
\text { of Engineering Education }\end{array}$ & & & 1 & & & 1 \\
\hline Sudáfrica & South African Journal of Education & & 1 & & & & 1 \\
\hline Total & & 18 & 62 & 18 & 5 & 5 & 108 \\
\hline
\end{tabular}

Nota: SJR = SCImago Journal Rank, Negritas = revistas de acceso abierto

Para citar este texto:

Cantú González, Valeria, Leonardo David Glasserman Morales y María Soledad Ramírez Montoya. 2019. "Comportamiento métrico sobre evaluación de la educación en emprendimiento”. Investigación Bibliotecológica: archivonomía, bibliotecología e información 33 (79): 99-117. http://dx.doi.org/10.22201/iibi.24488321xe.2019.79.57902 